\title{
Implementing Dynamic Mathematics Resources with GeoGebra at the College Level
}

\author{
doi:10.3991/ijet.v4i3.784 \\ Ljubica Dikovic \\ Business Technical College, Uzice, Serbia
}

\begin{abstract}
The dynamic nature of linear and vector algebra, differential calculus or integration, could be in conjunction with a dynamic/interactive method of display. Although GeoGebra provides multiple representations of mathematical objects, it can help students discover connections between mathematical objects and their graphical representation. Students take more responsibility for their own learning, and the dynamics of classroom activity shift to more discussion, interest, motivation to study, and cooperative learning. The contribution of this paper is to present new trends in technology and learning. In a technical sense, this paper presents a Geogebra applet, which could be especially important for the future development of e-learning of college mathematics. In a methodological sense, the contribution of this paper is to present a methodological framework with several specific examples. The aim of this paper to show how concepts of dynamic geometry can be applied to topics in calculus with suggested GeoGebra software tools. In addition, the work emphasizes the important role of free open-source software packages for mathematics teaching world-wide.
\end{abstract}

Index Terms - GeoGebra, college mathematics, FOSS

\section{INTEGRATION OF TECHNOLOGY IN THE}

\section{TRADITIONAL TEACHING OF COLLEGE MATHEMATICS}

The modern vision of education has relyied on the application of new communication and computer technologies with the aim of grasping new levels of creativity and knowledge in the context of the investigation.

ICTs and 'Computer Aided Teaching' have become important parts of contemporary life, and are widely used to improve teaching and learning techniques. The main forms of teaching mathematics with computers are:

- Web teaching and learning systems

Using ICTs, many Universities have established virtual education and distance learning systems in the field of mathematics, offering all new possibilities for students.

- Mathematics software packages

Many mathematics software packages have been developed, which are very powerful and have numerous functions, such as:

- Instantaneous numerical and symbolic calculations;

- Data collecting, analysis, exploration, and visualization;

- Modeling, simulation, and prototyping;

- Presentation graphics and animation in 2D and 3D;

- Application development.
The goal is to use technologies to provide an environment for active exploration of mathematical structures through multiple representations, or to show students some aspects of mathematics that are not possible with pen and paper. The students can concentrate on ideas; additionally computers can stimulate the students' geometric intuition through interesting visualizations in $2 \mathrm{D}$ or $3 \mathrm{D}$, or make it possible to ask questions involving theoretical issues that are arithmetically too complicated to be treated in a traditional way. [7]

\section{GEOGEBRA?}

Open Source Software / Free Software (OSS/FS or FOSS) are programs whose licenses give users the freedom to run the program for any purpose, to study and modify the program, and to redistribute copies of either the original or modified program.

Today, we can see various detailed explanations of the terms "Open Source Software" and "Free Software", but we note that those who use the term "Open Source Software" emphasize technical advantages of such software (e.g. better reliability and security), while those who use the term "Free Software" emphasize freedom (e.g. for control and use by other users).

The Open Source Initiative (OSI) is a non-profit organization formed to develop and educate about standards and benefits of open source systems, and to enable better communications among numerous open-source communities.

The distribution terms of open-source software must comply with the following criteria:

- The program must be freely distributed.

- The source code must be included.

- Anyone must be allowed to modify the source code.

- Modified versions can be redistributed again.

- The license must not require the exclusion of other software.

- Integrity source code of the author.

- No discrimination against persons or groups.

- No discrimination against fields of endeavor.

- Distribution of license. The rights attached to the program must apply to all to whom the program is redistributed without the need for an additional licenses.

- License must not restrict other software that is distributed along with the licensed software.

- License must be technology-neutral. [1], [2], [3], [4] 
In this moment, Geogebra is one of the most innovative open source math software available under the GNU General Public License. [6]

Geogebra works on a wide spectrum of platforms and operating systems which have installed Java virtual machine.

GeoGebra is free, educational, dynamic mathematics software for both teaching and learning mathematics from middle school through college and University level (see Hohenwarter \& Preiner, 2007). GeoGebra offers geometry, algebra and calculus features in a fully connected, compacted and easy-to-use software environment [9]. In other words, this tool extends concepts of dynamic geometry to the fields of algebra and mathematical analysis.

Specifically designed for educational purposes, GeoGebra can help students grasp experimental, problemoriented and discovery learning of mathematics both in classroom and at home.

Students can use simultaneously a computer algebra system and an interactive geometric system; so, in this way, they can significantly increase their cognitive abilities.

GeoGebra is a dynamic geometry software that supports constructions with points, lines and all conic sections. Also, it provides typical features of a Computer Algebra System such as finding important points of functions (roots, local extrema and inflection points of functions), directly capturing equations and coordinates, and finding derivates and integrals. That is the reason while the GeoGebra is a good choice for multiple representations of mathematic objects (See Figure 1). [5]

The basic idea of GeoGebra's interface is to provide two representations of each mathematical object in its algebra and graphics windows. If a user changes an object in one of these windows, its representation in the other one will be immediately updated.

To use a dynamic worksheet, students do not need GeoGebra installed on their computers. The only requirements are a Web browser and Java.

Also, advanced teachers can convert those dynamic, virtually worksheets into interactive exercises with GeoGebra's JavaScript interface and through setting applet parameters. This gives incredible flexibility; also it allows for the creaton of exercises with automatic feedback or exercises that are randomly generated.

For teachers, GeoGebra offers the powerful opportunity to create interactive, stimulating, online learning environments which encourages users to share free materials on the Internet.

Computer algebra systems (such as Mathematica, Maple, and so on, e.g.) and dynamic geometry software (such as Geometer's Sketchpad, Cabri Geometry, and so on, e.g.) are powerful technological tools for teaching mathematics. Numerous research results suggest that these software packages can be used to encourage discovery, experimentation and visualization in traditionally teaching of mathematics. However, researches suggest that, for the majority of teachers, solely providing technology is the problem for the successful integration of technology into teaching (Ruthven \& Hennessy 2004). [10]

In that sense, a suggested solution for applying technology in the college math's teaching is software packet GeoGebra.
The advantages of using GeoGebra are:

- In comparison to a graphing calculator, GeoGebra is more user-friendly.

- GeoGebra was created to help students gain a better understanding of mathematics. Students can manipulate variables easily by simply by dragging "free" objects around the plane of drawing, or by using sliders. Students can generate changes through manipulating techniques of the free objects, and then they can learn how the dependent objects will be affected. In this way, students have the opportunity to solve problems by investigating mathematical relations dynamically.

- Students can personalize their own creations through adaptation of interface (e.g. font size, language, quality of graphics, color, coordinates, line thickness, line style and other features).

- GeoGebra provides a good opportunity for cooperative learning. Students have the opportunity to learn in pairs or group, or to learn from one another.

- The algebra input allows users to generate new objects or to modify those already existing, by the command line. The worksheet files can easily be published as Web pages.

\section{GeoGebra is lacking in the following:}

- Students without previous programming experience will hardly enter algebraic commands in the input box. Although the basic commands are not difficult to learn, students may feel embarrassed or quite lost.

- Also, some methodological approaches such as telling students to "play with the variables" or "see what happens" is not adequate for many students.

- In a technical sense, Geogebra does not have in-built support for animation. So, putting modules for animating inside Geogebra should become an important technical element for future versions.

- Future extensions of the software GeoGebra will surely include more symbolic features of computer algebra systems which will further increase possible complex applications in the mathematical analyses.

\section{DEVELOPMENT OF THE INTERACTIVE TEACHING MATERIAL BY GEOGEBRA}

This short paper presents a few examples of computer uses of GeoGebra software in teaching differential calculus.

This software has changed situation in my classes, and has opened up new opportunities. Students have learned new techniques and have been able to use materials and options. They have also been able to use a variety of representations. Most important, students have felt more capable and familiar to solve numerous types of problems in Mathematics.

GeoGebra is a powerful development graphical environment used primarily to build mathematics objects and concepts easily, with minimal informatics experience required.

We could use GeoGebra to help our students better understand some complicated or very abstract math concepts. This software is amazing especially for visual learners, who can learn, explore, understand and practice some math concepts with those suggested tools. 
A dynamic GeoGebra worksheet is an interactive web page (html file) that consists of a dynamic figure (interactive applet) with corresponding explanations, questions, and tasks for students. When a user exports a construction from GeoGebra, both the graphics window and the algebra window will be shown on the dynamic worksheet. [11]

GeoGebra can be used for mathematical analyses in the following lectures:

- Definition of the graph of a function,

- Drawing graphs of elementary functions (e.g. linear, exponential, logarithmic, and trigonometric functions),

- Drawing graphs of transformations functions, after interactive transformations by using sliders or directly on the graph,

- Drawing graphs of complex functions,

- Important points of functions (roots, local extremes, inflection points),

- Definition of the limit of a function at a point,

- Concept of derivative as a limit secant line to tangent line at some point,

- Graphical relation between function of derivative, primitive function and integral,

- Concept of integral as a limit upper and lower Riemann sums

The following examples illustrate an overview of possible applications of GeoGebra for teaching basic concepts of calculus, as more critical and important abstractions can be taught later.

They are fully dynamic, meaning that points can be moved along function graphs, parameters are changeable using sliders, and text adapts automatically to changes.

Example: The worksheet "Derivative - Shape of a Slope Function" allows students to find the shape of the slope function of a trigonometric sin function by interactively modifying a dynamic figure.

The concept of derivative also potentially creates difficulties among students who lack proper understanding and visualization skills.

To motivate the cognitive transition from the picture of 'converges process secant line to tangent line' towards the derivative of some function, we could experimentally introduce the graph of the slope function (see Fig. 1).

The following applet demonstrates the derivative as a slope function.

The applet shows a movable point $T$ that lies on the function $f(x)=\sin x$.

Tangent $t$ is created for the point $\mathrm{T}$ with respect to $f(x)=\sin x$ as well as its slope $s$.

Then, point $B$ is defined as $B=(x(T), s)$.

When someone drags point $T$ along the function $f(x)=\sin x$, the trace of point $B$ shows the slope of that function for every $x$. This is the graph of our slope function.

Students can now try to find an equation for this slope function so that its graph fits the trace of $T$. Doing so; they are actually looking for the derivative of function (Figure 1).

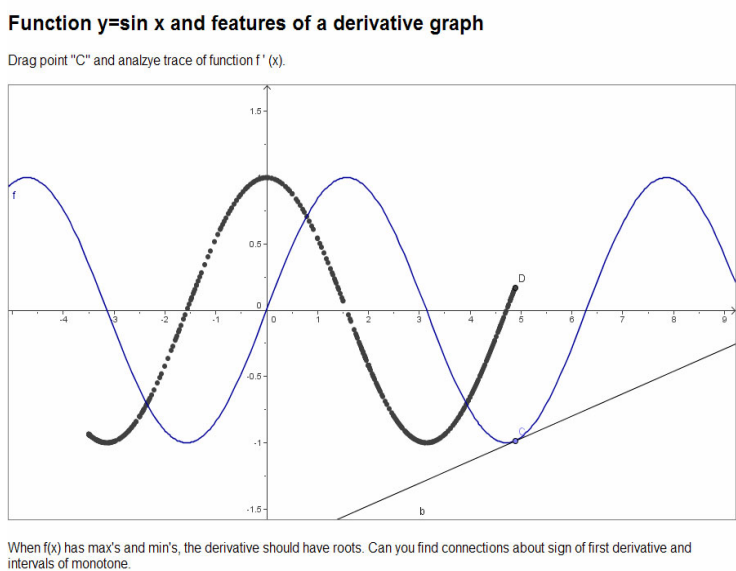

Ljubica Dikovic, 10.12.2008, Napravjeno programom GeoGebra

Figure 1. Exploring the connections between the initial function and its derivative

GeoGebra offers a command for the tangent to a function $f(x)=\sin x$, at $x=a$, for example:

$$
(a=2, f(x)=\sin x, t=\text { Tangent }[a, f]) \text {. }
$$

By animating a point $a$ (see Fig. 1) the tangent slides along the graph of function $f(x)=\sin x$. The tangent is changed dynamically too.

Function $f^{\prime}(x)=(\sin x)^{\prime}=\cos x$ is a derivative of function $f(x)=\sin x$.

$$
\text { Function } g(x)=\int f(x) d x=\int \sin x d x=\cos x,
$$
and its derivative is a function $f(x)=\sin x$,

$$
(g(x))^{\prime}=\left(\int f(x) d x\right)^{\prime}=\left(\int \sin x d x\right)^{\prime}=\cos x
$$

After that, students can check their assumption by typing the equation into GeoGebra's input field and comparing the corresponding function graph with the experimentally created trace of the slope function.

Since GeoGebra allows students to modify the initial function $f(x)$ any time, this applet can also be used to investigate different types of functions and their derivatives. Additionally, it can help students explore different rules for derivations, for example: Why does a constant term as the initial function have no impact on the derivative? (See Fig. 1)

The following applet explores relations between the derivative of function and the features of a function. In this way, students should be able to find the roots of the first derivative, points of local extremes, or conclude that monotone intervals are in connection with a sign of first derivative of that function (See Fig. 2)

The parameters of initial function $f(x)=\sin x$ can be modified using sliders allowing students to investigate the parameters' influence on the initial function, and to explore the connections between the initial function and its derivatives.

By using a worksheet with a teacher's assistance, students can systematically analyses the derivatives of different type of functions (See Fig. 3) 


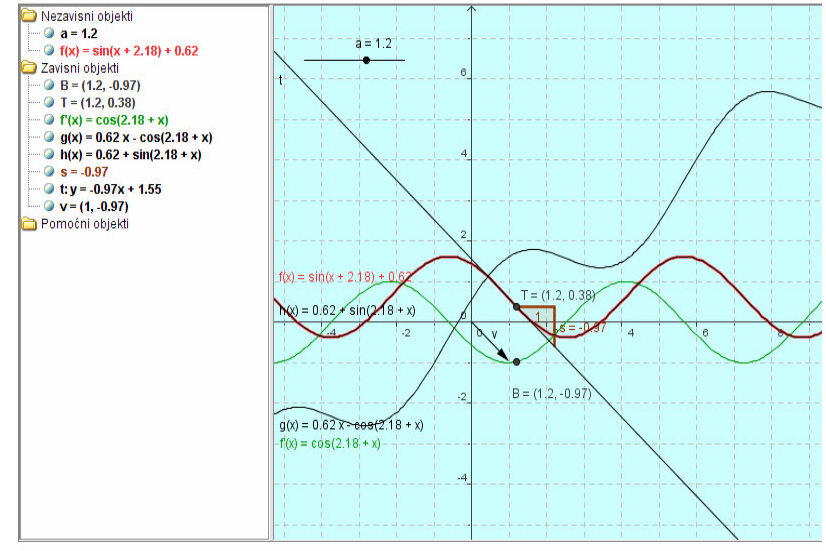

Ljubica Dikovic, 05.12.2008, Created with GeoGebra

Figure 2. Graphs of the functions $y^{\prime}=\sin x$ and $y^{\prime}=(\sin x)^{\prime}$ or tracing the slope function of $\sin (x)$.

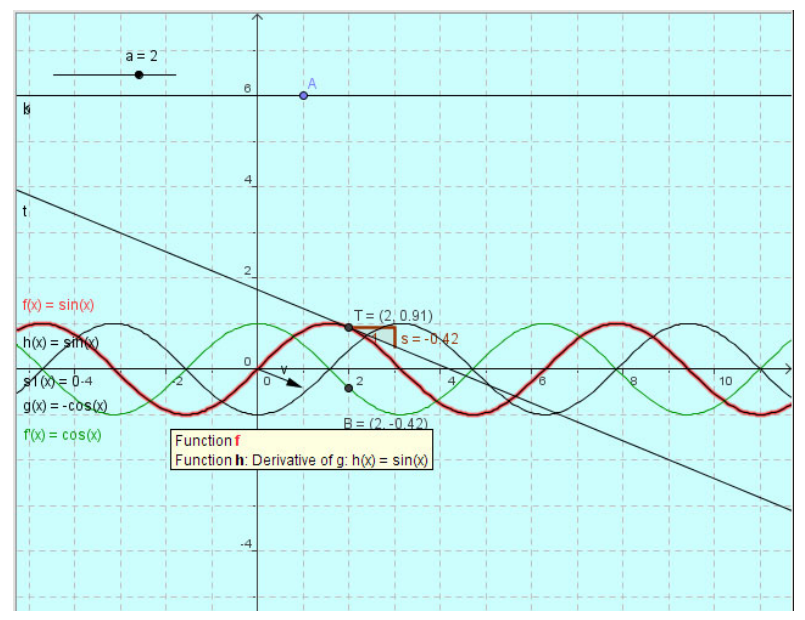

Figure 3. Exploring the connections between the initial function and its derivative and integral.

\section{CONCLUSION}

GeoGebra provides multiple representations of mathematical objects and can help students discover connections between mathematical objects and their graphical representation. The dynamic nature of linear and vector algebra, differential calculus or integration could be in a conjunction with dynamic/interactive method of display.

GeoGebra provides many possibilities to help students obtain an intuitive feeling and to adequately visualize math processes. The use of this software's tools allows students to explore a wider range of function types, and provides students the opportunity to make the connections between symbolic, visual, and numeric representations. Students should learn new techniques and should be able to model and evaluate a situation that is challenging, interesting, and real.

\section{REFERENCES}

[1] Dikovich Lj., „An Interactive Learning and Teaching of Linear Algebra by Web Technologies: Some Examples", Journal the Teaching of mathematics, Publisher: The Mathematical Society of Serbia, Beograd, ISSN: 1451-4966, Issue: X_2, Date: 2007, Pages: $109-116$

[2] NCTM (2000). Principles and Standards for School Mathematics. http://standards.nctm.org

[3] Hohenwarter, M.; Preiner, J., Dynamic Mathematics with GeoGebra, Journal for Online Mathematics and its Applications, Volume 7, March 2007, Article ID 1448

[4] K. Ruthven, S. Hennessy, S. Brindley, Teacher representations of the successful use of computer-based tools and resources in secondary-school English, mathematics and science, Teaching and Teacher Education, Volume 20, Issue 3, 2004, pages 259-275 (doi:10.1016/j.tate.2004.02.002)

[5] Hohenwarter, M.; Preiner, J., Taeil Yi, Incorporating GeoGebra into Teaching Mathematics at the College Level, Proceedings of ICTCM 2007, Boston, MA, available on address http://www.geogebra.org/publications/2007 ICTCM geogebra/

[6] http://opensource.org/

[7] http://www.dwheeler.com/oss_fs_why.html

[8] http://www.gnu.org/philosophy/categories.html

[9] http://www.geogebra.org

\section{AUTHOR}

Ljubica Dikovic is with the Business Technical College, Uzice, Serbia (e-mail: dikoviclj@gmail.com).

Submitted 25 December 2008. Published as resubmitted by the authors on 14 September 2009. 